\title{
EVALUACIÓN DE UN TERMOFOSFATO EN EL CRECIMIENTO Y PRODUCCION DE FRESA (Fragaria x ananassa Duch.) cv. 'ALBIÓN'
}

\section{EVALUATION OF THERMOPHOSPHATE IN GROWTH AND PRODUCTION THE STRAWBERRY (Fragaria x ananassa Duch.) cv. 'ALBIóN'}

\author{
Fabián Galindo-López ${ }^{1}$, Elberth Hernando Pinzón-Sandoval' ${ }^{2}$, Wilmer Alejandro Quintana-Blanco ${ }^{3}$, \\ Pablo Antonio Serrano ${ }^{4}$, Miguel Galán ${ }^{5}$
}

\begin{abstract}
${ }^{1}$ Ing. Agr., Grupo de Investigaciones Agrícolas (GIA). Universidad Pedagógica y Tecnológica de Colombia, e-mail: fadagalo@ hotmail.com; ${ }^{2}$ Ing Agr., M.Sc. Fisiología Vegetal. Universidad Pedagógica y Tecnológica de Colombia, e-mail: elberth.pinzon@uptc.edu.co; ${ }^{3}$ Ing. Agr., Grupo de Investigaciones Agrícolas (GIA). Universidad Pedagógica y Tecnológica de Colombia, e-mail: wilquintana61@hotmail.com; ${ }^{4}$ Ing Agr., M.Sc. Ciencias Ambientales. Universidad Pedagógica y Tecnológica de Colombia, e-mail: pablo.serrano@uptc.edu.co; Ing. Agr., Grupo GIPSO, Maestría en Fisiología Vegetal. Universidad Pedagógica y Tecnológica de Colombia, e-mail: magalanma@hotmail.com.
\end{abstract}

Rev. U.D.C.A Act. \& Div. Cient. 21(1): 61-69, Enero-Junio, 2018

https://doi.org/10.31910/rudca.v21.n1.2018.663

\section{RESUMEN}

La fresa es una de las frutas más importantes en el mundo, por sus múltiples usos industriales, medicinales y culinarios. En Colombia, la producción se concentra en los departamentos de Cundinamarca, Antioquia, Norte de Santander, Cauca y Boyacá. Su crecimiento y productividad están determinados por una adecuada fertilización en elementos, como nitrógeno $(\mathrm{N})$, potasio $(\mathrm{K})$, calcio $(\mathrm{Ca})$ y fósforo $(\mathrm{P})$, siendo el $\mathrm{P}$ un elemento de gran importancia, en diversos procesos fisiológicos, pero con baja disponibilidad para las plantas, en suelos con condiciones de acidez. Por esta razón, el objetivo de la investigación fue evaluar el efecto de una fuente no convencional de fósforo (termofosfato), en el crecimiento y en la producción de plantas de fresa cv. 'Albión', bajo condiciones del municipio de Viracachá, Boyacá. Se utilizó un diseño experimental completamente al azar, con 4 tratamientos, que correspondieron a dosis crecientes de termofosfato $\left(0,300,600\right.$ y $\left.900 \mathrm{~kg} \mathrm{ha}^{-1}\right)$, con 4 repeticiones. Se evaluó el contenido de clorofilas totales, peso seco de parte aérea y raíz, número de estructuras reproductivas, producción de frutos, concentración foliar de P, Ca y silicio (Si). Se presentaron diferencias significativas $(P \leq 0,05)$ en las variables clorofilas totales, peso seco de raíz, producción por calidad, concentración foliar de P y Si; las demás variables no mostraron diferencias estadísticas. Los mejores resultados, se presentaron con los tratamientos de 300 y $600 \mathrm{~kg} \mathrm{ha}^{-1}$ de termofosfato, convirtiéndolo en una alternativa de suministro en suelos con problemas de acidez y baja disponibilidad de P.
Palabras clave: Fósforo, nutrición mineral, acidez intercambiable, dinámica nutricional.

\section{SUMMARY}

The strawberry is one of the most important fruits in the world for its multiple industrial, medicinal and culinary uses. In Colombia, the production is concentrated in the departments of Cundinamarca, Antioquia, Norte de Santander, Cauca and Boyacá. Its growth and productivity are determined by an adequate fertilization in elements such as nitrogen $(\mathrm{N})$, potassium $(\mathrm{K})$, calcium $(\mathrm{Ca})$ and phosphorus $(\mathrm{P})$, being $\mathrm{P}$ an element of great importance in various physiological processes but with low availability for plants in soils with acidic conditions. For this reason, the aim of this study was to evaluate the effect of an unconventional source of phosphorus (thermophosphate), on the growth and production of strawberry plants cv. 'Albión' under conditions of the municipality of Viracachá, Boyacá. A completely randomized experimental design was used, with 4 treatments, which were increasing doses of thermophosphate $(0,300$, 600 and $900 \mathrm{~kg} \mathrm{ha}^{-1}$ ) with 4 repetitions. The content of total chlorophylls, dry weight of aerial part and root, number of reproductive structures, production and foliar concentration of phosphorus, calcium and silicon were evaluated. There were significant differences $(P \leq 0.05)$ in the variables total chlorophyll, root dry weight, production by quality, foliar concentration of $\mathrm{P}, \mathrm{Ca}$ and silicon $(\mathrm{Si})$; the other variables did not show statistical differences. The best results were 
presented with the treatments of 300 and $600 \mathrm{~kg} \mathrm{ha}^{-1}$ of thermophosphate turning it into an alternative supply in soils with acidity problems and low availability of $P$.

Key words: Phosphorus, mineral nutrition, interchangeable acidity, thermophosphate.

\section{INTRODUCCIÓN}

La fresa (Fragaria $x$ ananassa Duch.) es uno de los frutos más apreciados en el mundo, debido a sus excelentes características de aroma, de color, de textura, posee compuestos bioactivos, como vitamina $\mathrm{C}$ y K, filoquinona, folato y constituyentes fenólicos, con capacidad antioxidante y puede ser consumida, tanto en fresco como procesada (Giampieri et al. 2012; Khoshnevisan et al. 2013). Esta especie es cultivada en países ubicados, tanto en la zona tropical como templada del mundo, principalmente, en el continente europeo y americano (Galvão et al. 2017). En Colombia, en el 2016, se produjeron 61.468t de fresa, siendo Cundinamarca el departamento con mayor producción, con una participación de 41.425t, seguido por Antioquia, con 11.179t; Norte de Santander, con 3.713t; Cauca, 2.330t y Boyacá, con 1.413t (Agronet, 2018). En los últimos años, el cultivo de fresa ha alcanzado un notable desarrollo, siendo mayor al de especies similares, como la mora y se debe a que es una fruta que se puede cultivar durante todo el año, dinamizando las economías locales, por la alta demanda de mano de obra, a través de todo el ciclo de cultivo.

La fresa presenta un rápido crecimiento, influenciado por condiciones, como luz, salinidad del suelo, calidad del agua, temperatura, fitosanidad y disponibilidad de nutrientes, siendo este último, determinante para satisfacer la demanda fotosintética y el crecimiento adecuado de la planta (Li et al. 2010). La calidad de la fresa depende de una adecuada fertilización, en donde los nutrientes requeridos, en mayor medida, son: nitrógeno, potasio, calcio y fósforo, este último, con gran importancia en la fase de establecimiento y de floración (Gaskell, 2014). Las plantas toman el fósforo como fosfato inorgánico; sin embargo, es uno de los macronutrientes menos disponibles en el suelo, ya que su disponibilidad se ve limitada, debido a su alta reactividad con los metales, junto con factores, tales como el $\mathrm{pH}$ y la composición del suelo (Plaxton \& Lambers, 2015).

El fósforo $(\mathrm{P})$ es un macronutriente esencial para todos los seres vivos, forma parte de estructuras biológicas, participa en procesos celulares y su ausencia repercute negativamente en el crecimiento y en el desarrollo de las plantas (Bernal et al. 2007). Además, es uno de los diecisiete elementos esenciales que cumple funciones estructurales en macromoléculas y transfiere energía en procesos metabólicos de biosíntesis y de degradación (Marschner, 2012).
La fertilización en el cultivo de fresa, se debe realizar a lo largo de su ciclo de crecimiento, destacándose tres escenarios, en los que la fertilización es esencial: crecimiento vegetativo, floración y fructificación; sin embargo, la fertilización se ve limitada en zonas tropicales y subtropicales, en donde existen grandes extensiones de suelos ácidos y de baja fertilidad, por presencia de altas concentraciones de aluminio, que genera la precipitación del fósforo, reduciendo las concentraciones en el suelo (Fernández, 2007). Por esta razón, los suelos requieren de la aplicación de fertilizantes fosforados, para alcanzar altos niveles de productivida; no obstante, la mayor parte del fósforo aplicado es rápidamente fijado en el suelo, evitando ser absorbido por las raíces de las plantas y provocando severos trastornos fisiológicos (Fernández, 2007).

Los fertilizantes fosfatados son materiales cuya composición, en términos de porcentaje, es relativamente elevado en fósforo $\left(\mathrm{P}_{2} \mathrm{O}_{5}\right)$; existe una amplia gama de fertilizantes y de enmiendas fosfóricas, que aportan fósforo a los suelos, uno de ellos es el termofosfato o fosfato térmico, conocido como material fosfórico, sometido a elevadas condiciones de temperatura (Almeyda \& Niño, 2010). Este material, contiene concentraciones de calcio y de silicio, provenientes de los materiales que componen la roca fosfórica, de la cual, se obtiene, estimulando la absorción de fósforo, principalmente, en suelos de condición ácida (Fernández, 2007). La roca fosfórica, al ser sometida a un tratamiento térmico, rompe su estructura rígida para obtener un producto con solubilidad intermedia, mejorando la disponibilidad de fósforo a corto y largo plazo y ofreciendo a la planta un mayor porcentaje de P, en forma asimilable, durante todo su ciclo fenológico (Fernández \& Noguera, 2003). Según Quintana-Blanco et al. (2017), la aplicación de un fosfato térmico en condiciones de un suelo moderadamente acido, favoreció, de manera significativa, variables, como contenido total de clorofilas, masa seca de órganos, área foliar, fósforo en tejido foliar y componentes de rendimiento, en plantas de fríjol arbustivo cv 'ICA Cerinza'.

Por lo anterior, el objetivo de esta investigación fue evaluar el efecto del termofosfato sobre el crecimiento y la producción de plantas de fresa (Fragaria x ananassa Duch.) cv. 'Albión', como alternativa de manejo de la fertilización fosfórica, en zonas productoras de fresa del departamento de Boyacá.

\section{MATERIALES Y MÉTODOS}

La investigación, se llevó a cabo, en su fase de campo, durante el segundo semestre de 2016, en la finca El Naranjo, vereda los Naranjos, municipio de Viracachá, Boyacá, a una altura de 2.520 m s.n.m. y una temperatura promedio de $15^{\circ} \mathrm{C}$. La fase de laboratorio, se realizó en el laboratorio de fisiología vegetal de la Universidad Pedagógica y Tecnológica de Colombia, con sede en Tunja, Boyacá, ubicada al norte 
del municipio, con coordenadas $5^{\circ} 32^{\prime 2} 25^{\prime \prime} \mathrm{N} 73^{\circ} 21^{\prime} 41^{\prime \prime} \mathrm{O}$. Se realizó un análisis de suelo (Tabla 1), a partir del cual, se determinaron los tratamientos.
Se empleó un diseño experimental completamente al azar (DCA), con 4 tratamientos y 4 repeticiones, para un total de 16 unidades experimentales (UE); cada UE estuvo conformada por 5 esquejes certificados provistos por Proplantas ${ }^{\circledR}$ del

Tabla 1. Propiedades físico-químicas del suelo utilizado en el experimento.

\begin{tabular}{|c|c|c|}
\hline Propiedad & Valor & Interpretación \\
\hline $\mathrm{pH}$ & 4,12 & Extremadamente Ácido (Castro, 1998) \\
\hline $\mathrm{MO}(\%)$ & 3,5 & Bajo \\
\hline $\mathrm{P} \mathrm{Bray} \mathrm{II}(\mathrm{ppm})$ & 27,6 & Medio \\
\hline $\mathrm{Al}\left(\mathrm{cmol} \mathrm{kg}^{-1}\right)$ & 1,1 & Medio \\
\hline $\mathrm{Ca}\left(\mathrm{cmol} \mathrm{kg}^{-1}\right)$ & 3,22 & Medio \\
\hline $\mathrm{Mg}\left(\mathrm{cmol} \mathrm{kg}^{-1}\right)$ & 0,87 & Bajo \\
\hline $\mathrm{K}\left(\mathrm{cmol} \mathrm{kg}^{-1}\right)$ & 0,58 & Alto \\
\hline Textura $(\%)$ & A: $27 ; \mathrm{L:} 35 ; \mathrm{Ar}: 38$ & Franco Arcillosa \\
\hline
\end{tabular}

cultivar 'Albión', los cuales, provienen de proceso de estratificación en cuarto frío, a una temperatura de $-1^{\circ} \mathrm{C}$ y humedad relativa del $90 \%$, durante un periodo de 6 meses; estos fueron aclimatados a temperatura ambiente de $14^{\circ} \mathrm{C}, 24 \mathrm{~h}$ antes de la siembra. La siembra, se realizó bajo condiciones de campo, en una cama protegida con cubierta plástica de tipo Guardián, Proplantas ${ }^{\circledR}$. Los tratamientos fueron T1: Testigo sin aplicación; T2: 300 $\mathrm{kg} \mathrm{ha}^{-1}$; T3: $600 \mathrm{~kg} \mathrm{ha}^{-1}$ y T4: $900 \mathrm{~kg} \mathrm{ha}^{-1}$ de termofosfato. Como fuente de termofosfato, se empleó un producto comercial, con una composición garantizada de $\mathrm{P}_{2} \mathrm{O}_{5}$ : 20\%; $\mathrm{CaO}: 28 \%$; $\mathrm{MgO}$ : 8,5\%; $\mathrm{SiO}_{2}: 15 \%$. Los tratamientos, se aplicaron al momento de la siembra y a los 60 días después de la siembra (dds).

La siembra, se realizó ubicando los estolones en sitios ya marcados dentro de la cama, en los que, previamente, se aplicaron los tratamientos, con una distancia de $0,5 \mathrm{~m}$ entre surco y 0,3m entre plantas, para una densidad de 66.666 plantas ha ${ }^{-1}$. Posteriormente, se procedió a realizar el manejo agronómico, que consistió en la ejecución de labores culturales, como fertilización y control fitosanitario del cultivo.

A los 60 días después de trasplante (ddt), momento en el que las plantas presentaron como mínimo tres hojas verdaderas, se inició a medir el contenido total de clorofilas, con un medidor SPAD 502 plus, en intervalos de 15 días; en dichas mediciones, se tomaron 10 lecturas. Se cuantificó el número de estructuras reproductivas (estolones) por planta, se realizó conteo a los $60 \mathrm{ddt}$.
A los 130 dds, las plantas presentaron un buen desarrollo vegetativo (presencia de 4 coronas por planta), se determinó la masa seca de raíz y parte aérea, mediante balanza electrónica Acculab VIC 612, de 0,01g de precisión, luego de secado de las muestras, en estufa Memmert, a $85^{\circ} \mathrm{C}$, hasta que se alcanzó peso constante (72 horas).

A los 130 ddt, cuando se estabilizó la producción de frutos en las plantas, se procedió a medir las variables de productividad, como: número de frutos por planta, realizando los respectivos conteos en los días de cosecha (cada 8 días por un mes); la producción, se clasificó por calidades comerciales, para lo cual, se midió el diámetro de los frutos en el plano ecuatorial, con ayuda de un pie de rey; posteriormente, se clasificó la fruta, de acuerdo con las calidades comerciales presentadas en la Norma Técnica Colombiana para fresa (NTC 4103). Para determinar contenido de fósforo, calcio y silicio, se cosecharon hojas del tercio bajo medio y alto de la planta hasta obtener una muestra de $80 \mathrm{~g}$ de tejido seco (aproximadamente, $300 \mathrm{~g}$ de tejido fresco). El contenido de fósforo y de calcio en tejido vegetal, se determinó a través del método de calcinación, a $600^{\circ} \mathrm{C}$; digestión ácida y valoración, por espectrofotometría visible y el contenido de silicio en tejido vegetal, se realizó por digestión vía húmeda cerrada, en horno microondas y cuantificación por absorción atómica-técnica de llama, en el laboratorio AGRILAB®.

Los datos obtenidos fueron sometidos a pruebas de normalidad y de homogeneidad de varianza, mediante las pruebas 
de Shapiro-Wilk y Levene, respectivamente; comprobados los supuestos, se realizó análisis de varianza. Las variables que mostraron diferencias estadísticas fueron sometidas a pruebas de comparación de medias de Tukey $(\mathrm{P} \leq 0,05)$. Los análisis se realizaron con el programa estadístico SAS v.9.2e (SAS Institute Inc., Cary, NC).

\section{RESULTADOS Y DISCUSIÓN}

El contenido de clorofila total presentó diferencias entre los tratamientos $(P \leq 0,05)$ en la semana 6 y 16 ddt. El tratamiento que presentó el mayor valor en clorofila total (SPAD) fue el de $300 \mathrm{~kg} \mathrm{ha}^{-1}$ del termofosfato comercial, durante la mayor parte del experimento, seguido de la aplicación de $900 \mathrm{~kg} \mathrm{ha}^{-1}$, con valores a la semana $16 \mathrm{ddt}$, de 48,25 $\pm 1,74 \mathrm{y}$
47,97 $\pm 0,39$ unidades SPAD, respectivamente. Los menores valores los presentó el tratamiento testigo, con 42,75 $\pm 1,2$ unidades SPAD, en la semana 16 ddt (Figura 1).

Pinzón-Sandoval et al. (2017) indican que la aplicación de fertilizantes con contenidos de $\mathrm{Mg}$, como el termofosfato empleado en el estudio, aumenta el contenido de clorofila total, debido a que este elemento es componente fundamental de la molécula de clorofila, haciendo que el proceso fotosintético sea eficiente.

Los resultados encontrados en el presente estudio concuerdan con lo reportado por Estrada-Ortiz et al. (2011), quienes indican que la aplicación del 30\% de fósforo, en forma de fosfitos en plantas de fresa cv. 'Festival', generó un aumento

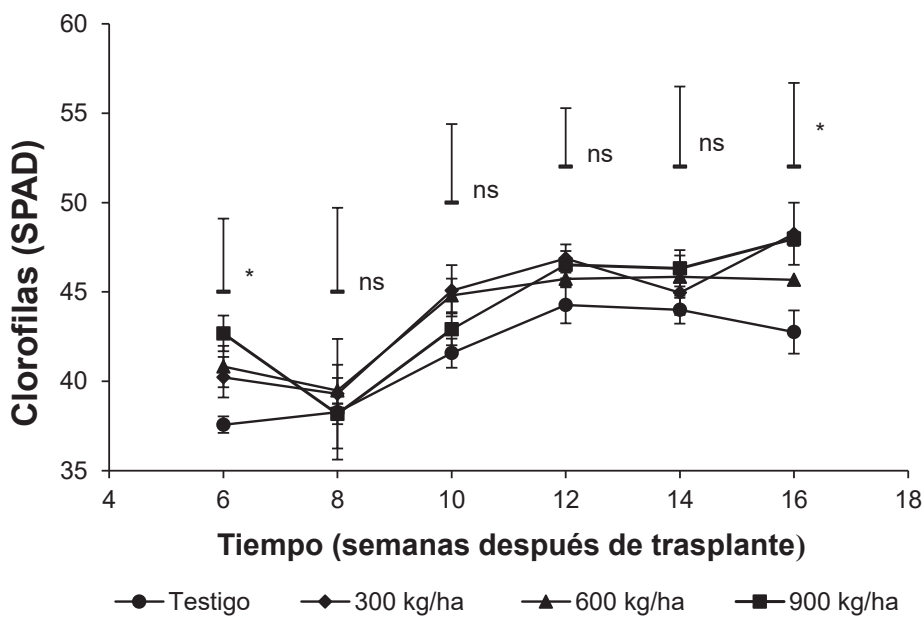

Figura 1. Comportamiento de las clorofilas totales en fresa cv 'Albión', sometido a diferentes dosis de termofosfato. Barras verticales en los tratamientos indican error estándar $(n=4)$. Barras sobre cada punto de muestreo indica la diferencia mínima significativa, según la prueba de Tukey $(\mathrm{P} \leq 0,05)$, ns: no existen diferencia estadística, *: diferencias significativas.

en la concentración de clorofila a, b y total, en etapa de fructificación. Por su parte, Bojović \& Stojanović (2006) indican que el fósforo, como elemento nutriente, tiene influencia sobre la estabilidad de la molécula de clorofila. Latsague et al. (2014) reportan que los contenidos de clorofila a, b y total fueron influenciados con un aumento significativo en hojas de Berberidopsis corallina, por la aplicación de P, en dosis de $150 \mathrm{mg} \mathrm{L}^{-1}$ y su interacción con otros elementos, como el nitrógeno; además, estos elementos constituyen diferentes proteínas, enzimas y moléculas de transferencia de energía, como clorofila, ADP y ATP, las cuales, son de gran importancia en el proceso de fotosíntesis.

El peso seco de la parte aérea (PSA) no presentó diferencias estadísticas entre tratamientos, mientras que la variable peso seco de raíz (PSR) sí presentó diferencias significativas
$(\mathrm{P} \leq 0,05)$ (Figura 2A). El mayor valor para PSA lo obtuvo la aplicación de $300 \mathrm{~kg} \mathrm{ha}^{-1}$ de termofosfato, con 56,39 $\pm 2,97 \mathrm{~g}$ y, para PSR, lo presentó el tratamiento de $600 \mathrm{~kg} \mathrm{ha}^{-1}$, con un valor de 33,52 $\pm 3,11 \mathrm{~g}$.

Aunque no hubo diferencias significativas para el PSA, se observó un incremento de peso seco en los tratamientos de $300 \mathrm{~kg} \mathrm{ha}^{-1}$ y $600 \mathrm{~kg} \mathrm{ha}^{-1}$ de termofosfato frente al testigo. Nkaa et al. (2014) indican que el peso seco de las raíces y la materia seca total en Vigna unguiculata mostraron resultados positivos, en respuesta a la aplicación de fósforo. Según Estrada-Ortiz et al. (2011), la partición de materia seca es un indicador de la distribución de fotoasimilados hacia los distintos puntos de demanda en crecimiento, por lo que el peso seco real corresponde a la acumulación de dichos fotoasimilados, en las diferentes partes de las plantas. 
A

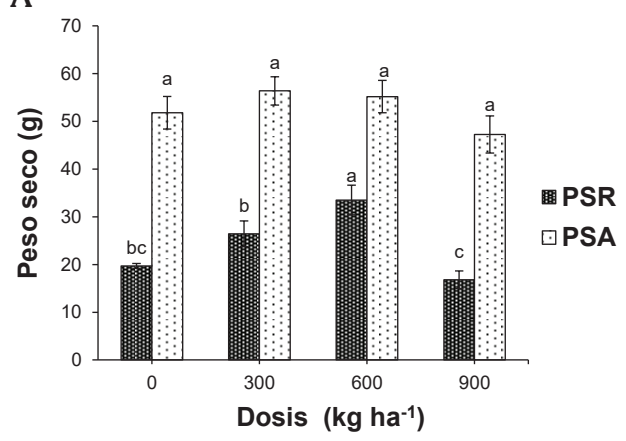

B

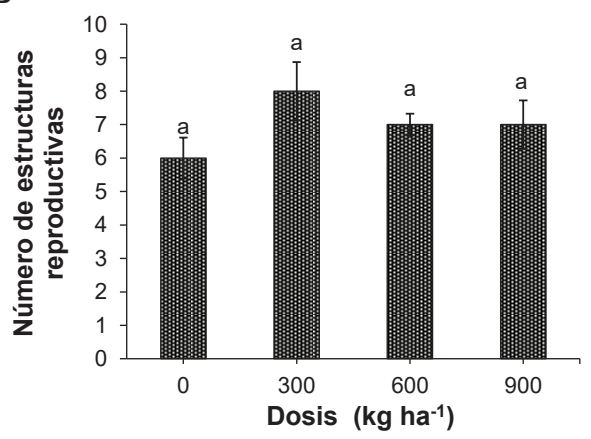

Figura 2. A. Peso seco aéreo (PSA), Peso seco raíz (PSR). B. Número de estructuras reproductivas; en plantas de fresa cv 'Albión', sometido a diferentes dosis de fosfato térmico. Tratamientos seguidos de letras distintas presentan diferencias significativas, según la prueba de Tukey $(P \leq 0,05)$, barras verticales indican error estándar $(n=4)$.

Para el caso del tratamiento de $900 \mathrm{~kg} \mathrm{ha}^{-1}$ de termofosfato, se obtuvo una disminución de materia seca aérea (Figura 2A) y se debe, probablemente, a la pérdida de follaje, como consecuencia del agotamiento sufrido por la formación de los frutos y por la lenta asimilación de otros nutrientes como el Ca y Si, ocasionada, quizá, por el exceso de P, el cual, es considerado como elemento antagónico del $\mathrm{Ca}$ y $\mathrm{Si}$, cuando es suministrado en altas cantidades (Molina et al. 1993). Una deficiencia de P, se manifiesta por retrasos en la floración y la baja producción de frutos y semillas, pero caso contrario, un exceso puede provocar la fijación de otros elementos, como el zinc, en el suelo, repercutiendo negativamente en el crecimiento de las plantas (García, 2010).

El aumento de materia seca, tanto en parte aérea como en la raíz, se debe a que el fósforo participa, en gran medida, en la producción y en el transporte de fotoasimilados, a partir de la fotosíntesis (Estrada-Ortiz et al. 2011). El mismo autor indica que la partición de materia seca es un indicador de la distribución de fotoasimilados hacia los distintos puntos de demanda en crecimiento, por lo que el peso seco real corresponde a la acumulación de dichos fotoasimilados, en las diferentes partes de las plantas.

La variable número de estructuras reproductivas (estolones), no presentó diferencias significativas entre tratamientos $(P \geq 0,05)$ (Figura 2B). El mayor valor lo presentó la aplicación de $300 \mathrm{~kg} \mathrm{ha}^{-1}$ del termofosfato comercial, con una media de $8 \pm 0,61$ estolones, mostrando un aumento del $33 \%$ (2 estolones), en comparación al tratamiento testigo.

El P juega un papel importante en todos los procesos metabólicos que participan en la transferencia de energía, dentro de los que se encuentra la formación de hojas, de flores, de frutos y de órganos reproductivos, como los estolones; esto se pudo observar en la presente investigación, en donde el fosfato térmico utilizado participó activamente en la formación de estolones, considerada como una variable de gran importancia, a nivel de rendimiento. Medeiros et al. (2015) indican que la fresa es una especie en la que la nutrición fosfórica es muy importante, ya que influye sobre variables de crecimiento, número y peso de frutos, como también en el número y en el desarrollo de hojas, que son favorecidos por suministro de altos niveles de este nutrimento.

Para la variable producción, se presentaron diferencias significativas en cuanto a la calidad extra, según la norma NTC 4103, mientras que, para la calidad primera, segunda y producción total, no se presentaron diferencias $(P \geq 0,05)$ (Figura 3). La media más alta para calidades extra, primera y segunda la presentaron los tratamientos 300 y $600 \mathrm{~kg} \mathrm{ha}^{-1}$ de termofosfato, respectivamente. En cuanto a la producción total, el mejor comportamiento se observó con la aplicación de $300 \mathrm{~kg}$ $\mathrm{ha}^{-1}$, con una media de $477,8 \pm 39,72 \mathrm{~g} / \mathrm{planta} / \mathrm{mes}$, incluyendo calidad extra, primera y segunda, aumentando en un $32 \%$ la producción, en comparación con el tratamiento testigo.

Li et al. (2014) encontraron que la aplicación de nitrógeno y de fósforo en diferentes dosis aumentó el número de frutos, respuesta que asociaron a que el fósforo es uno de los nutrientes que más requieren las plantas para su desarrollo, formando compuestos relacionados a la base genética de la planta y componentes energéticos del metabolismo vegetal, como ATP y ADP, necesarios como fuente de energía, en procesos metabólicos para la formación de frutos. Romero et al. (2012) encontraron que, tratamientos aplicados en fresa de fertilización mineral con $\mathrm{P}$, registraron los valores más altos para diámetro ecuatorial y polar, variable fundamental en la clasificación de fresa por calidades comerciales.

El fosfato térmico utilizado en el estudio aporta nutrientes, como P, $\mathrm{Ca}, \mathrm{Mg}$ y Si, elementos de gran importancia para la 


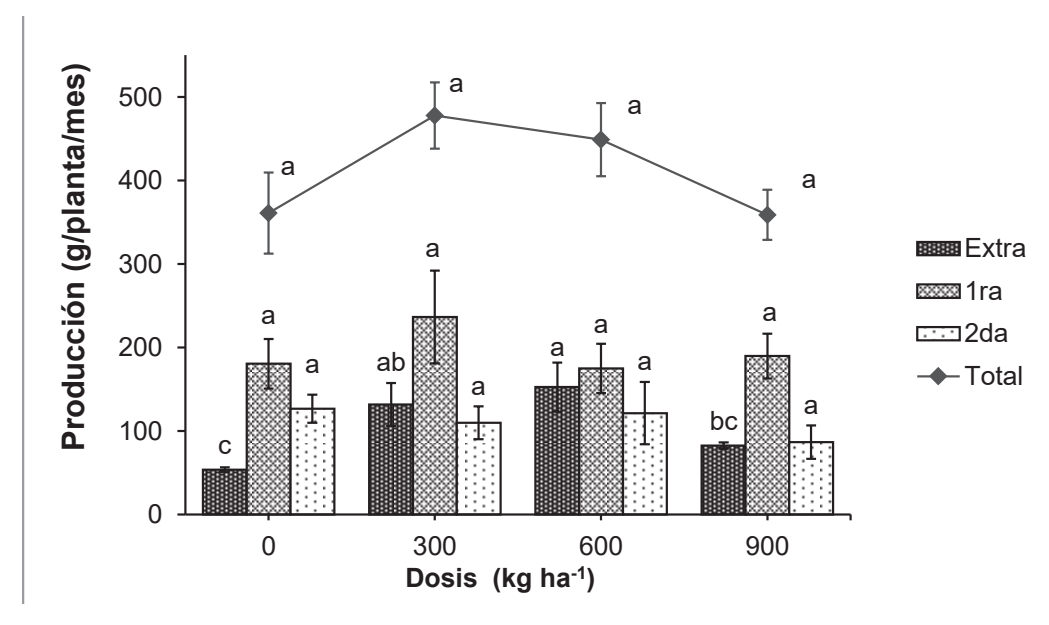

Figura 3. Producción por calidades y total de frutos de fresa cv 'Albión', sometido a diferentes dosis de fosfato térmico. Tratamientos seguidos de letras distintas presentan diferencias significativas, según la prueba de Tukey $(P \leq 0,05)$, barras verticales indican error estándar $(n=4)$.

planta (Pinzón-Sandoval et al. 2017). El Ca es un elemento esencial en el desarrollo de diferentes órganos en las plantas, ya que es indispensable para la formación y la multiplicación de las células y en la determinación de la calidad de los frutos (Taiz \& Zeiger, 2010). El Mg es un elemento que forma parte de la molécula de clorofila, siendo clave en la fotosíntesis y en la producción de fotoasimilados, necesarios en la formación de órganos y de estructuras (Taiz \& Zeiger, 2010). El Mg es particularmente importante para las plantas; en estas, un $75 \%$ se encuentra en las hojas y está involucrado en la síntesis de proteínas y entre un $15-20 \%$ de Mg total, se asocia con pigmentos, como las clorofilas (White \& Broadley, 2009), Por su parte, Marschner (2012) indica que el Mg participa como regulador del equilibrio catión-anión en las células; Gerendás \& Führs (2013) afirman que este elemento está vinculado dentro de los procesos de regulación osmótica junto con el K y Hermans et al. (2013) argumentan que el Mg actúa como un cofactor de una serie de enzimas involucradas en la fijación de carbono fotosintético y metabolismo celular.

Para la variable concentración foliar de fósforo (\%), se presentaron diferencias significativas entre tratamientos $(P \leq 0,05)$. La mayor concentración la presentó la aplicación de $300 \mathrm{~kg}$ $\mathrm{ha}^{-1}$, con un valor de $0,3 \pm 0,03 \%$; el tratamiento testigo y la aplicación de $600 \mathrm{~kg} \mathrm{ha}^{-1}$ presentaron un comportamiento intermedio (Figura 4).

El fósforo es un elemento esencial para las plantas y se requiere un adecuado suplemento de este nutriente para que la planta crezca y se reproduzca, de una manera óptima (Munera Vélez \& Meza Sepulveda, 2012). Según Marschner (2012), en la mayoría de plantas, la concentración de fósforo, que se considera adecuada, es $0,2 \%$, en relación al peso seco; en el presente estudio, obtuvo una mayor acumulación de fósforo, a nivel foliar, que puede tener un efecto directo en la capacidad fotosintética de las plantas (Singh et al. 2013).

Estos resultados indican que el uso de un fosfato térmico generó un aumento de la concentración en tejido de fósforo, representando un incremento en el crecimiento y en la productividad de fresa cv 'Albión`, en la dosis adecuada, ya que la concentración de fósforo en los fosfatos térmicos presenta una condición citrosoluble e hidrosoluble, ideal para condiciones de suelos ácidos, teniendo una disponibilidad, de este elemento, durante todo el ciclo fenológico de la planta (Fernández \& Noguera, 2003).

Para la concentración foliar de calcio no se presentaron diferencias significativas entre tratamientos $(P \geq 0,05)$ (Figura $5 \mathrm{~A})$. El tratamiento que presentó el mayor valor fue la aplicación de $900 \mathrm{~kg} \mathrm{ha}^{-1}$ de termofosfato, con $2,05 \pm 0,25 \%$ y el menor el tratamiento testigo, con una media de $1,64 \pm 0,09 \%$.

El calcio es considerado un constituyente importante de las paredes celulares con movimiento vía corriente transpiratoria (Marschner, 2012). Por otra parte, el calcio es un nutriente de naturaleza estructural, pues hace parte del componente de las paredes y membranas celulares, razón por la cual, es indispensable su presencia para la formación de nuevas células (Larcher, 2003).

De acuerdo con la revisión adelantada por Larcher (2003), la concentración de calcio en la masa seca de las especies cultivadas varía entre 0,04 y 1,3\%. En los resultados obtenidos, se tienen concentraciones más altas que las reportadas en la literatura, relacionándolo, específicamente, con la toma de 


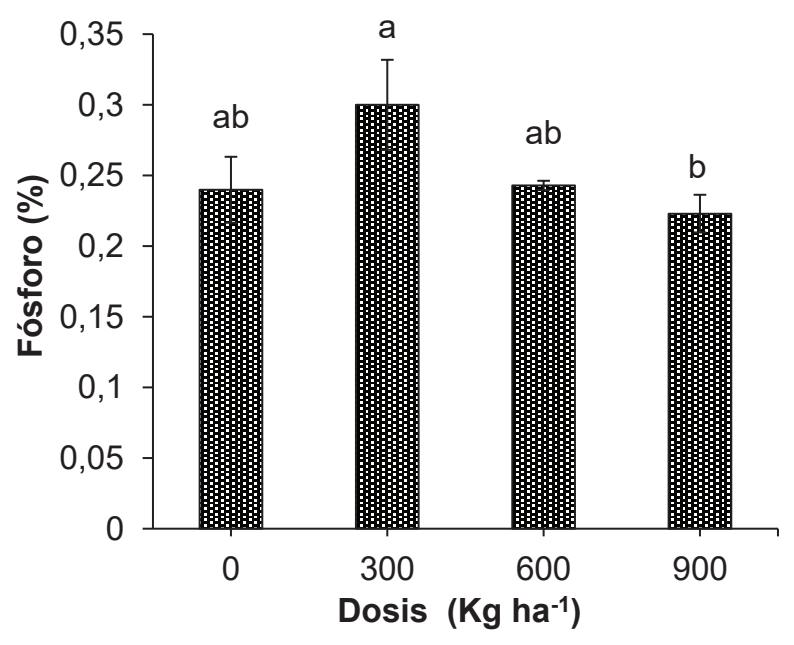

Figura 4. Concentración foliar de fósforo (\%) en plantas de fresa cv 'Albión', sometido a diferentes dosis de fosfato térmico. Tratamientos seguidos de letras distintas presentan diferencias significativas, según la prueba de Tukey $(P \leq 0,05)$, barras verticales indican error estándar $(n=4)$.

fósforo, debido a que concentraciones excesivas de calcio, en materia seca de las plantas, puede ocasionar una disminución en la absorción de fósforo, lo que se observa en la aplicación de 600 y $900 \mathrm{~kg} \mathrm{ha}^{-1}$.

Para la concentración foliar de silicio, se presentaron diferencias significativas entre tratamientos $(P \leq 0,05)$. La aplicación de 600 y $900 \mathrm{~kg} \mathrm{ha}^{-1}$ presentaron las mejores concentraciones del elemento, con medias de 1,26 $\pm 0,12$ y $1,21 \pm 0,05 \%$, respectivamente. El tratamiento control y la aplicación de $300 \mathrm{~kg} \mathrm{ha}^{-1}$ no presentaron diferencias significativas entre sí, pero sí frente a la aplicación que mostró el mayor valor (Figura 5B).

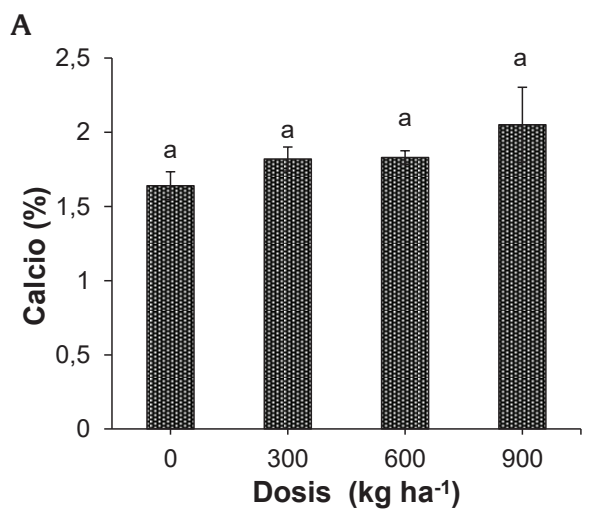

El silicio es el segundo elemento mineral más abundante en la corteza terrestre después del oxígeno; su contenido en las plantas es variable y depende fundamentalmente de la especie, oscilando entre el 0,1 y 10\%, del peso seco de las plantas superiores (Epstein, 1999). El silicio ha sido reconocido por aumentar la eficiencia en la captación de la luz solar (Yoshida et al. 1969).

Según la especie, las plantas acumulan cierta cantidad de silicio en sus tejidos; las gramíneas, especialmente, son las que mayor contenido de este elemento acumulan y las especies dicotiledóneas, no se benefician en un gran porcentaje de $\mathrm{Si}$, por lo cual, fertilizantes silicatados, altamente solubles, proporcionarían una mayor absorción de este elemen-

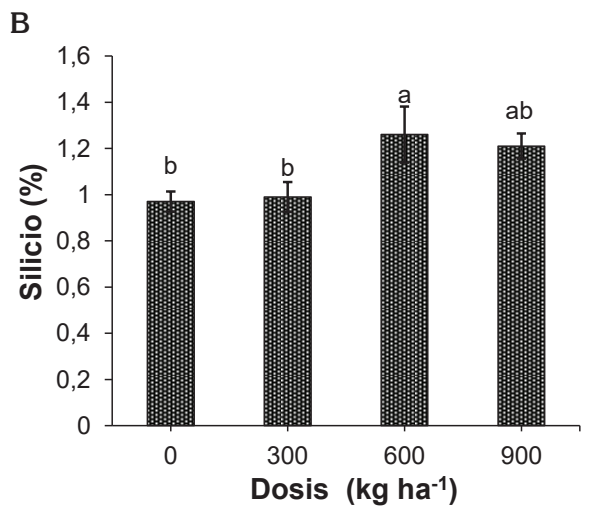

Figura 5. A. Concentración foliar de calcio (\%). B. Concentración foliar de silicio en plantas de fresa cv 'Albión', sometido a diferentes dosis de fosfato térmico. Tratamientos seguidos de letras distintas presentan diferencias significativas, según la prueba de Tukey $(P \leq 0,05)$, barras verticales indican error estándar $(n=4)$. 
to, mostrando sus efectos benéficos en las plantas (Epstein, 1999). Además, el silicio contribuye a mejorar la absorción de fósforo en condiciones de suelo de la zona tropical e intertropical, principalmente ácidos, ya que en la solución del suelo este elemento desplaza óxidos e hidróxidos de $\mathrm{Al}$ y Fe, desfijando el fósforo y suministrándolo a la planta.

En fresa, se encontró una acumulación de silicio mayor al $1 \%$, indicando que esta planta es acumuladora del elemento, con un modo pasivo de absorción, lo que indica que lo toma de la solución a una velocidad similar de absorción del agua (Mitani \& Ma, 2005) y que se beneficia de todos los aportes.

Las aplicaciones de termofosfato influyeron positivamente en variables de crecimiento en plantas de fresa (Fragaria $x$ ananassa Duch.) cv 'Albión', como: contenido total de clorofilas, peso seco, tanto de parte aérea como de raíz, encontrando que los mejores resultados se obtuvieron con las aplicaciones de 300 y $600 \mathrm{~kg} \mathrm{ha}^{-1}$, del termofosfato comercial. Para las variables de rendimiento, como número de estolones y de productividad (peso de frutos y calidades comerciales), los mejores resultados fueron obtenidos con el tratamiento de $300 \mathrm{~kg} \mathrm{ha}^{-1}$.

Agradecimientos: La presente Investigación, se realizó con apoyo de Cales y Derivados Calcáreos Río Claro Naranjo y Compañía S.C.A. Conflicto de Intereses: El manuscrito fue preparado y revisado con la participación de todos los autores, quienes declaramos que no existe conflicto de interés que ponga en riesgo la validez de los resultados presentados.

\section{BIBLIOGRAFÍA}

1. AGRONET. 2016. Cifras estadísticas cultivo de fresa. Disponible desde Internet en: http://www.agronet. gov.co/estadistica/Paginas/default.aspx (con acceso 14/05/2018).

2. ALMEYDA, A.; NIÑO, R. 2010. Obtención de abono enriquecido a partir de roca fosfórica y Leonardita mediante procesos piro metalúrgicos y químicos. Universidad Industrial de Santander. 66p.

3. BERNAL, L.; COELLO, P.; ACOSTA, J.; MARTÍNEZ, E. 2007. Efecto de la deficiencia de fósforo en el metabolismo de carbono de plántulas de fríjol (Phaseolus vulgaris L.). Agrociencia. 41(4):417-423.

4. BOJOVIĆ, B.; STOJANOVIĆ, J. 2006. Some wheat leaf characteristics in dependence of fertilization. Kragujevac J. Science. 28:139-146.

5. CASTRO, H. 1998. Fundamentos para el conocimiento y manejo de los suelos agrícolas. Instituto Universitario Juan de Castellanos. 360p.
6. EPSTEIN, E.1999. Silicon. Annual Review of Plant Physiology and Plant Molecular Biology. 50:641-664.

7. ESTRADA-ORTIZ, E.; TREJO, L.; GÓMEZ, F.; NÚĨ̃̃EZ, R.; SANDOVAL, M. 2011. Respuestas bioquímicas en fresa al suministro de fósforo en forma de fosfito. Rev. Chapingo. Serie Horticultura. 17(3):129-138.

8. FERNÁNDEZ, M. 2007. Fósforo: amigo o enemigo. ICIDCA. Sobre los Derivados de la Caña de Azúcar. 41(2):51-57.

9. FERNÁNDEZ, S.; NOGUERA, R. 2003. Producción de fosfatos térmicos a partir de rocas fosfóricas nacionales. Agronomía Tropical; 53(1):49-58.

10. GALVÃO, A.; RESENDE, L.; MALUF, W.; RESENDE, J.; VILELA DE FERRAZ, A.; MARODIN, J. 2017. Breeding new improved clones for strawberry production in Brazil. Acta Scientiarum. Agronomy. 39(2):149155.

11. GARCÍA, M. 2010. Guía técnica del cultivo de la papaya. CENTA, Centro nacional de tecnología agropecuaria y forestal. Disponible desde Internet en: http://www. centa.gob.sv/docs/guias/frutales/GUIA\%20CULTIVO\%20PAPAYA.pdf (con acceso 05/01/2018).

12. GASKELL, M. 2014. Factores que guían las prácticas de fertilización para la producción de fresa convencional y orgánica en California. 4th International Plant Nutrition Symposium. University of California Cooperative Extension. p.1-44.

13. GERENDÁS, J.; FÜHRS, H. 2013. The significance of magnesium for crop quality, Plant Soil. 368:101128.

14. GIAMPIERI, F.; TULIPANI, S.; ÁLVAREZ-SUÁREZ, J.; QUILES, J.; MEZZETTI, B.; BATTINO, D. 2012. The strawberry: composition, nutritional quality, and impact on human health. Nutrition. 28(1):9-19.

15. HERMANS, C.; CONN, S.; CHEN, J.; XIAO, Q.; VERBRUGGEN, N. 2013. An update on magnesium homeostasis mechanisms in plants, Metallomics. 5:1170-1183.

16. KHOSHNEVISAN, B.; RAFIEE, S.; MOUSAZEDH, $\mathrm{H}$. 2013. Enviromental impact assessment of open field and greenhouse strawberry production. Eur. J. Agron. 50:29-37. 
17. LATSAGUE, M.; SÁENZ, P.; MORA, M. 2014. Efecto de la fertilización con nitrógeno, fósforo y potasio, sobre el contenido foliar de carbohidratos, proteínas y pigmentos fotosintéticos en plantas de Berberidopsis corallina Hook.f. Gayana Bot. 71(1):17-28.

18. LARCHER, W. 2003. Physiological plant ecology; Ecophysiology and stress physiology of functional groups. Fourth edition. Springer. 513p.

19. LI, H.; HUANG, R.; LI, T.; HU, K. 2010. Ability of nitrogen and phosphorus assimilation of seven strawberry cultivars in a northern Atlantic coastal soil. In: $19^{\text {th }}$ World Congress of Soil Science, Soil Solutions for a Changing World. Brisbane, Australia. p.1-6.

20. Li, H.; Li, T.; Fu, G.; Hu, K. 2014. How strawberry plants cope with limited phosphorus supply: Nursery-crop formation and phosphorus and nitrogen uptake dynamics. Journal of Plant Nutrition and Soil Science. 177:260-270.

21. MARSCHNER, P. 2012. Mineral nutrition of higher plants. 3rd. Ed. Elsevier. Oxford, UK. 645p.

22. MEDEIROS, R.; PEREIRA, W.; RODRIGUES, R.; NASCIMENTO, R.; SUASSUNA, J.; DANTAS, T. 2015. Growth and yield of strawberry plants fertilized with nitrogen and phosphorus. Rev. Bras. Eng. Agríc. Ambient. 19:865-870.

23. MITANI, N.; MA, J. 2005. Uptake system of silicon in different plant species. J. Experimental Botany. 56:1255-1261.

24. MOLINA, E.; SALAS, R.; CASTRO, A. 1993. Curva de crecimiento y absorción de nutrimentos en fresa (Fragaria $x$ ananassa cv. Chandler) en Alajuela. Rev. Agronomía Costarricense. 17(1):67-73.

25. MUNERA VELEZ, G.A.; MEZA SEPULVEDA, D.C. 2012. El fosforo elemento indispensable para la vida vegetal. Universidad Tecnológica de Pereira. 52p.

26. NKAA, F.A.; NWOKEOCHA, O.; IHUOMA, O. 2014. Effect of Phosphorus fertilizer on growth and yield of cowpea (Vigna unguiculata). J. Pharmacy and Biological Sciences. 9(5):74-82.

27. PINZÓN-SANDOVAL, E.; QUINTANA-BLANCO, W.; CELY-REYES, G. 2017. Effect of magnesium silicate in cv. 'ICA Cerinza' common bean (Phaseolus vulgaris L.) under field conditions. Rev. Fac. Nac. Agron. 70(3):8285-8293.

28. PLAXTON, W.; LAMBERS, H. 2015. Phosphorus Metabolism in Plants. Elsevier. Plant Physiol. 193:45-53.

29. QUINTANA-BLANCO, W.; PINZÓN-SANDOVAL, E.; TORRES, D. 2017. Efecto de un fosfato térmico sobre el crecimiento y producción de fríjol (Phaseolus vulgaris L.) CV ICA CERINZA. Rev. U.D.C.A Act. \& Div. Cient. 20(1):51-59.

30. ROMERO, C.; OCAMPO, J.; SANDOVAL, E.; TOBAR, J. 2012. Fertilización orgánica-mineral y orgánica en el cultivo de fresa (Fragaria $x$ ananassa Duch.) bajo condiciones de invernadero. Rev. Ra Ximhai. 8(3):41-49.

31. SINGH, S.; BADGUJAR, G.; REDDY, V.; FLEISHER, D.; TIMLIN, D. 2013. Effect of phosphorus nutrition on growth and physiology of cotton under ambient and elevated carbon dioxide. J. Agron. Crop Sci. 199:436-448.

32. TAIZ, L.; ZEIGER, E. 2010. Plant. Physiology. $5^{\text {th }}$ ed. Sinauer Associates Inc., Publishers Sunderland, Massachusetts U.S.A. 778p.

33. WHITE, P.; BROADLEY, M. 2009. Biofortification of crops with seven mineral elements often lacking in human diets - iron, zinc, copper, calcium, magnesium, selenium and iodine. New Phytol. 182:49-84.

34. YOSHIDA, S.; NAVASERO, S.; RAMIREZ, E. 1969. Effects of silica and nitrogen supply on some leaf characters of rice plants. Plant Soil. 31:48-56.

Recibido: Febrero 2 de 2018

Aceptado: Mayo 31 de 2018

\section{Cómo Citar:}

Galindo-López, F.; Pinzón-Sandoval, E.H.; Quintana-Blanco, W.A.; Serrano, P.A.; Galán, M. 2018. Evaluación de un termofosfato en el crecimiento y producción de fresa (Fragaria $x$ ananassa Duch.) cv. 'Albión'. Rev. U.D.C.A Act. \& Div. Cient. 21(1): 61-69. 